\title{
New relations for correlation functions in Navier-Stokes turbulence
}

\author{
Gregory Falkovich ${ }^{1}$, Itzhak Fouxon ${ }^{2}$, and Yaron $\mathrm{Oz}^{2}$ \\ 1 Physics of Complex Systems, Weizmann Institute of Science, Rehovot 76100, Israel. \\ 2 Raymond and Beverly Sackler School of Physics and Astronomy, \\ Tel-Aviv University, Tel-Aviv 69978, Israel
}

\begin{abstract}
We consider the steady-state statistics of turbulence in the inertial interval. The Kolmogorov flux relation (4/5-law) is shown to be a particular case of the general relation on the current-density correlation function. Using that, we derive an analogous flux relation for compressible turbulence and a new exact relation for incompressible turbulence.
\end{abstract}

PACS numbers: 


\section{INTRODUCTION}

The Kolmogorov flux relation is a rare exact analytic result one has for the correlation functions of the velocity $\boldsymbol{v}$ in the Navier-Stokes equation. It is traditionally derived considering quadratic invariants like kinetic energy in an incompressible flow (or squared vorticity in two dimensions). Conservation of the kinetic energy, $\int v^{2} / 2$, by an unforced Euler equation means that one can define the energy flux in $k$-space and write the continuity equation for the energy spectral density [12]:

$$
\begin{aligned}
& \Pi_{k}=-\frac{1}{8 \pi^{2}} \int d^{3} r \frac{\sin (\mathbf{k} \cdot \mathbf{r})}{r} \frac{\partial}{\partial r_{i}}\left[\frac{r_{i}}{r^{2}} \frac{\partial}{\partial r_{j}}\left\langle u_{j} u^{2}\right\rangle\right] . \\
& \frac{\partial\left\langle\left|v_{k}\right|^{2}\right\rangle}{2 \partial t}+\operatorname{div} \Pi_{k}=0, \boldsymbol{u}(\boldsymbol{r}) \equiv \boldsymbol{v}(\boldsymbol{r})-\boldsymbol{v}(0) .
\end{aligned}
$$

Stationarity under the action of a large-scale force and small-scale viscosity means the constancy of the energy flux $\Pi_{k}$ over intermediate wavenumbers [12, 16, 18]:

$$
\nabla_{i}\left\langle u_{i} u^{2}\right\rangle=-4 \nabla_{i}\left\langle v_{i}(\boldsymbol{r}) v_{j}(\boldsymbol{r}) v_{j}(0)\right\rangle=-4 \epsilon
$$

That can also be written for the third moment of the longitudinal velocity difference, $u_{l}=$ $(\mathbf{u} \cdot \mathbf{r} / r)$ :

$$
\left\langle u_{l}^{3}\right\rangle=-12 \epsilon r / d(d+2)
$$

Here $d$ is the space dimensionality. For $d=3$ one obtains $\left\langle u_{l}^{3}\right\rangle=-4 \epsilon r / 5$, which is why the flux relation is sometimes called 4/5-law. Analogous relations are derived for the passive scalar turbulence, magnetized and helical flows [2, 4, 14, 15, 23, 25, 26, 27, 29]. If, however, the respective conservation law is non-quadratic (as the energy in compressible turbulence, for instance) then it is believed that the analogs of (2) are absent. In addition, there are no analogs of this relation for velocity moments of the orders $n$ different from three. Experiments demonstrate that $\left\langle u_{l}^{n}\right\rangle \propto r^{\zeta_{n}}$ with the scaling exponents $\zeta_{n}$, which are larger than one but smaller than $n / 3$, see e.g. [10, 12].

In this letter, we re-interpret the Kolmogorov relation in terms of currents and densities of the conserved quantities, which allows us to derive an analog for a compressible case and a new fundamental relation for an incompressible case. 


\section{GENERAL RELATION}

Consider a general class of classical field dynamics,

$$
\partial_{t} q^{a}+\nabla \cdot \boldsymbol{j}^{a}=f^{a}
$$

where $q^{a}, a=1, \ldots, N$ are densities, $\boldsymbol{j}^{a}$ are currents and $f^{a}$ are the external random source fields. These equations describe local conservation laws and provide a canonical form for the effective hydrodynamic description of a slow evolution of large-scale perturbations. Since the zero wave-number component of the density is conserved, low wave-number components evolve slowly by continuity, see e.g. [13]. The equations are closed via a constitutive relation that expresses currents in terms of the densities as a series in gradients:

$$
j_{i}^{a}=F_{i}^{a}(\{\rho\})+\sum_{j b} G_{i, j b}^{a}(\{\rho\}) \nabla_{j} \rho^{b}+\ldots
$$

where dots stand for higher order terms involving more derivatives. The zero-order, reactive, term leads to a conservative dynamics while the first-order term describes dissipation. For fluid mechanics, consideration of higher order terms in Eq. (5) is usually unnecessary so we limit ourselves to the following general class, which also contains the Navier-Stokes equation:

$$
\partial_{t} q^{a}+\frac{\partial F_{i}^{a}}{\partial r_{i}}=f^{a}-\frac{\partial}{\partial r_{i}}\left(\sum_{j b} G_{i, j b}^{a}(\{\rho\}) \nabla_{j} \rho^{b}\right) .
$$

We assume the standard mathematical formulation of the problem of turbulence where the forcing term $f^{a}$ is random and its statistics is stationary, spatially homogeneous and isotropic [12]. This implies that the same properties hold for the steady state statistics of $q^{a}$. The correlation length of the force will be denoted below by $L$.

The derivation of the Kolmogorov type relation for Eq. (6) proceeds as follows. Consider the steady-state condition $\partial_{t}\left\langle q^{a}(0, t) q^{a}(\boldsymbol{r}, t)\right\rangle=0$ (no summation over $a$ ). Employing the dynamical equation (6) and using statistical symmetries, one finds

$$
\begin{aligned}
& 0=\partial_{t}\left\langle q^{a}(0, t) q^{a}(\boldsymbol{r}, t)\right\rangle=-2 \frac{\partial}{\partial r_{i}}\left\langle q^{a}(0, t) F_{i}^{a}(\boldsymbol{r}, t)\right\rangle \\
& +2\left\langle q^{a}(0, t) f^{a}(\boldsymbol{r}, t)\right\rangle-\frac{\partial}{\partial r_{i}}\left\langle q^{a}(0, t)\left(\sum_{j b} G_{i, j b}^{a}(\{\rho\}(\boldsymbol{r}, t)) \nabla_{j} \rho^{b}(\boldsymbol{r}, t)\right)\right\rangle .
\end{aligned}
$$

We consider the limit of large correlation length $L$ of the forcing. That allows one to consider $r$ much smaller than $L$ yet still large enough, so that the last term becomes negligible as 
containing higher order of spatial derivatives. Because $r \ll L$ we have $f^{a}(\boldsymbol{r}, t) \approx f^{a}(0, t)$ and $\left\langle q^{a}(0, t) f^{a}(\boldsymbol{r}, t)\right\rangle \approx\left\langle q^{a}(0, t) f^{a}(0, t)\right\rangle \equiv \epsilon_{a}$, where the constant $\epsilon_{a}$ is the mean input rate of $\left(q^{a}\right)^{2}$. Hence we obtain for the single-time correlation function

$$
\nabla_{i}\left\langle q^{a}(0) F_{i}^{a}(\boldsymbol{r})\right\rangle=\epsilon_{a} .
$$

Assuming in addition isotropy one finds

$$
\left\langle q^{a}(0) F_{i}^{a}(\boldsymbol{r})\right\rangle=\frac{\epsilon_{a} r_{i}}{d}
$$

\section{PARTICULAR CASES}

A simple example is the passive scalar turbulence when some substance with the density $q$ and diffusivity $\kappa$ is carried by a flow with the velocity $\boldsymbol{v}$. The current is $\boldsymbol{j}=q \boldsymbol{v}-\kappa \nabla q$. The flux (Yaglom) relation is as follows: $\langle q(0) q(\boldsymbol{r}) \boldsymbol{v}(\boldsymbol{r})\rangle=\epsilon \boldsymbol{r} / d$.

Another example is the turbulence of a barotropic fluid where the pressure $p(\rho)$ is a function of the fluid density $\rho$ only. In this case, $\mathbf{q}=(\rho \mathbf{v}, \rho), F_{j}^{i}=\rho v_{i} v_{j}+p(\rho) \delta_{i j}$ and $\boldsymbol{j}^{4}=\rho \boldsymbol{v}$. The equations have the form

$$
\begin{aligned}
& \partial_{t} \rho+\nabla \cdot(\rho \boldsymbol{v})=0, \quad \partial_{t}\left(\rho v_{i}\right)+\partial_{j}\left(\rho v_{i} v_{j}+p \delta_{i j}\right) \\
& =-\partial_{j}\left[G_{j, k b}^{i}(\{\rho\}) \nabla_{k} \rho^{b}\right]+f^{i}
\end{aligned}
$$

where we took into account that $\boldsymbol{j}^{4}=\rho \boldsymbol{v}$ is exact to all orders. The source often has the form $f^{i}=\rho \nabla_{i} \Phi$ that corresponds to an external potential $\Phi$ (the analysis below can be easily generalized to other types of forcing as well). The exact form of $G_{j, k b}^{i}$ is not important below but its presence is necessary for a steady state. Indeed, the rhs of Eq. (10) breaks the energy conservation; the steady state holds due to the balance of the forcing that pumps fluctuations into the system and the dissipation that erases them. Now we use the general relation (8) to derive the new relation for the compressible turbulence described by Eqs. (9)(10). The application of the relation to $q^{4}=\rho$ with $\epsilon_{4}=0$ gives $\left\langle\rho(0, t) \rho(\boldsymbol{r}) v_{i}(\boldsymbol{r})\right\rangle=$

0. In fact this result holds for any relation between $r$ and $L$. Indeed, the steady-state condition $\partial_{t}\langle\rho(0, t) \rho(\boldsymbol{r}, t)\rangle=0=\partial_{i}\left\langle\rho(0) \rho(\boldsymbol{r}) v_{i}(\boldsymbol{r})\right\rangle$ applied to the general (isotropic) form $\left\langle\rho(0) \rho(\boldsymbol{r}) v_{i}(\boldsymbol{r})\right\rangle=A(r) r_{i}$, gives $A=C r^{-d}$, so that regularity at the origin requires $C=0$.

Application of Eq. (8) to $q^{j}$ with $j=1,2,3$ gives a non-trivial relation

$$
\sum_{j}\left\langle\rho(0) v_{j}(0)\left[\rho(\boldsymbol{r}) v_{j}(\boldsymbol{r}) v_{i}(\boldsymbol{r})+p(\boldsymbol{r}) \delta_{i j}\right]\right\rangle=\frac{\epsilon r_{i}}{d}
$$


where $\epsilon$ is defined in this case as $\langle\rho(0) \boldsymbol{v}(0) \cdot \boldsymbol{f}(0)\rangle=\epsilon$ (we summed over $j$ to get a more symmetric result). To the best of our knowledge, the formula (11) is new and presents a desired analog of Kolmogorov relation for the compressible turbulence see e. g. [21]. Probably, this relation was not derived before because it demands considering the steadystate condition for the fourth-order correlation function $\left\langle\rho(0, t) v_{j}(0, t) \rho(\boldsymbol{r}, t) v_{j}(\boldsymbol{r}, t)\right\rangle$, while usually in trying to find Kolmogorov type relations one considers steady state conditions for the second moment, like in magnetohydrodynamics by [25]. Note in passing that one can choose $q$ as the energy density and obtain yet another relation analogous to (15) below.

In the incompressible limit, $\rho=$ const and $\nabla \cdot \boldsymbol{v}=0$, the pressure term is zero; again, since it is a divergence-free vector that must be regular at the origin [see 22]. In this case, (11) is reduced to (2),

$$
\left\langle v_{i}(\boldsymbol{r}) v_{j}(\boldsymbol{r}) v_{j}(0)\right\rangle=\epsilon r_{i} / d,
$$

and Eq. (3) is implied. Hence (11) is indeed a general form of the Kolmogorov relation for an arbitrary Mach number. As we see, from a general viewpoint, the relation follows from the stationarity of the pair correlation function of the momentum density rather than from the energy spectral density. Indeed, $\epsilon$ in (11) is the input rate of the squared momentum and not that of the energy, which coincide (up to the factor $1 / 2$ ) only in the incompressible case.

We have shown how the Kolmogorov relation exploits the momentum conservation. Now in the same way we shall exploit the energy conservation and derive a new fundamental relation for incompressible turbulence. Energy conservation in the incompressible case means that the Navier-Stokes equation can be written as a continuity equation for the kinetic energy:

$$
\frac{\partial}{\partial t} \frac{v^{2}}{2}=-\frac{\partial}{\partial r_{i}}\left[v_{i}\left(\frac{v^{2}}{2}+p\right)\right]+\boldsymbol{f} \cdot \boldsymbol{v}+\nu v_{i} \nabla^{2} v_{i} .
$$

That means that one can choose $q=v^{2}$ which turns the general form (8) into a fifth-order relation. The only difference from the third-order relation (12) is that the pressure term is now nonzero. Note first that the condition $\partial\left\langle v^{4}\right\rangle / \partial t=0$ gives the single-point pressurevelocity correlation function:

$$
\left\langle v^{2} v_{i} \nabla_{i} p\right\rangle=\left\langle\boldsymbol{f} \cdot \boldsymbol{v} v^{2}\right\rangle+\nu\left\langle v^{2} v_{i} \nabla^{2} v_{i}\right\rangle .
$$


For the different-point moment, Eq. (8) gives

$$
\frac{\partial}{\partial r_{i}}\left\langle v_{i}(\boldsymbol{r})\left[\frac{v^{2}(\boldsymbol{r})}{2}+p(\boldsymbol{r})\right] v^{2}(0)\right\rangle=\left\langle\boldsymbol{f}(\boldsymbol{r}) \cdot \boldsymbol{v}(\boldsymbol{r}) v^{2}(0)\right\rangle+\nu\left\langle v^{2}(0) v_{i}(\boldsymbol{r}) \nabla^{2} v_{i}(\boldsymbol{r})\right\rangle .
$$

This relation can be further simplified by decoupling small-scale and large-scale fields. Force and velocity are large-scale fields that change over the scale $L$ so that one can put $\langle\boldsymbol{f}(\boldsymbol{r})$. $\left.\boldsymbol{v}(\boldsymbol{r}) v^{2}(0)\right\rangle=\left\langle\boldsymbol{f} \cdot \boldsymbol{v} v^{2}\right\rangle$ at $r \ll L$ as we did in deriving (8). Velocity differences and derivatives are small-scale fields that change respectively over the scale $r$ and the viscous length $\eta$ see e. g. [12]. In particular, the local energy dissipation, $\nu v_{i}(\boldsymbol{r}) \nabla^{2} v_{i}(\boldsymbol{r})$, is a small-scale field with the correlation radius $\eta$. When the distance $r$ is in the inertial interval, $r \gg \eta$, one can decouple large-scale and small-scale fields: $\nu\left\langle v^{2}(0) v_{i}(\boldsymbol{r}) \nabla^{2} v_{i}(\boldsymbol{r})\right\rangle \approx-\epsilon\left\langle v^{2}\right\rangle$. We also denote $\boldsymbol{V}=\boldsymbol{v}(\boldsymbol{r})+\boldsymbol{v}(0)$ and present

$$
\begin{aligned}
& 4\left\langle v_{i}(\boldsymbol{r}) v^{2}(\boldsymbol{r}) v^{2}(0)\right\rangle=-\left\langle u_{i}\left[v^{2}(\boldsymbol{r})-v^{2}(0)\right]^{2}\right\rangle=-\left\langle u_{i}(\boldsymbol{u} \cdot \boldsymbol{V})^{2}\right\rangle \\
& \approx-\left\langle u_{i} u^{2}\right\rangle\left\langle V^{2}\right\rangle\left\langle\cos ^{2} \alpha\right\rangle=-2\left\langle v^{2}\right\rangle\left\langle u_{i} u^{2}\right\rangle=8\left\langle v^{2}\right\rangle \epsilon \boldsymbol{r} / d
\end{aligned}
$$

Here $\alpha$ is the angle between $\boldsymbol{u}$ and $\boldsymbol{V}$ and in the second line we assumed that the vectors $\boldsymbol{u}$ and $\boldsymbol{V}$ are weakly correlated and can be decoupled when the distance $r$ is in the inertial interval, $r \ll L$. In the last equality we used the Kolmogorov relation (2). We thus find that in the inertial range, $\eta \ll r \ll L$, Eq. (15) is reduced to

$$
\frac{\partial}{\partial r_{i}}\left\langle v_{i}(\boldsymbol{r}) p(\boldsymbol{r}) v^{2}(0)\right\rangle=\left\langle\boldsymbol{f} \cdot \boldsymbol{v} v^{2}\right\rangle-2 \epsilon\left\langle v^{2}\right\rangle \equiv D
$$

We thus obtain the fundamental relation on the pressure-velocity correlation function which is the counterpart of the Kolmogorov relation (3):

$$
\left\langle\boldsymbol{v}(\boldsymbol{r}) p(\boldsymbol{r}) v^{2}(0)\right\rangle=D \boldsymbol{r} / d
$$

That relation can be tested experimentally and numerically. From a formal viewpoint, (11,17) are particular cases of the Hopf equations which express the stationarity of the correlation functions. Hopf equations are generally not closed; they impose some relations between different structure functions but do not allow to find them see e. g. [30]. On the contrary, our relations (11]17) allow one to find the correlation functions that are fluxes. Taking divergence of (18) gives $\left\langle v^{2}(0) v_{i}(\boldsymbol{r}) \nabla_{i} p(\boldsymbol{r})\right\rangle=D$ in the inertial interval, compare it with the single-point expression (14). 
Inverting the incompressibility condition, $\Delta p=-\nabla_{i} v_{j} \nabla_{j} v_{i}$, one expresses pressure:

$$
p(\boldsymbol{r})=\frac{1}{(d-2) \sigma_{d}} \int \frac{d \boldsymbol{r}^{\prime}}{\left|\boldsymbol{r}-\boldsymbol{r}^{\prime}\right|^{d-2}} \frac{\partial^{2} u_{i}\left(\boldsymbol{r}^{\prime}\right) u_{j}\left(\boldsymbol{r}^{\prime}\right)}{\partial r_{i}^{\prime} \partial r_{j}^{\prime}}, \quad \sigma_{d}=\frac{2 \pi^{d / 2}}{\Gamma(d / 2)},
$$

where $u_{i}\left(\boldsymbol{r}^{\prime}\right)=v_{i}\left(\boldsymbol{r}^{\prime}\right)-v_{i}(0)$. That allows one to present (18) as an integral relation on the fifth-order three-point moment of the velocity field (we denote $\hat{r}^{\prime}=\boldsymbol{r}^{\prime} / r^{\prime}$ ):

$$
\frac{1}{\sigma_{d}} \int \frac{d \boldsymbol{r}^{\prime}}{r^{\prime d}}\left\langle v^{2}(\boldsymbol{r}) \boldsymbol{v}(0)\left[u^{2}\left(\boldsymbol{r}^{\prime}\right)-d\left[\boldsymbol{u}\left(\boldsymbol{r}^{\prime}\right) \cdot \hat{r}^{\prime}\right]^{2}\right]\right\rangle=D \boldsymbol{r} / d .
$$

We expressed it via the differences in the square brackets to make it explicit that the integrand is regular at the origin.

Note that the force term in (17) is the input rate of the squared energy which for a general force statistics cannot be expressed via the energy input rate. In the particular case of a white Gaussian force, such expression is possible: $\left\langle f_{i} v_{i} v^{2}\right\rangle=(1+2 / d) \epsilon\left\langle v^{2}\right\rangle$ and $D=(2 / d-1) \epsilon\left\langle v^{2}\right\rangle$. Remark that the direct energy cascade, which is studied here, is absent for $d=2$. For $2 \mathrm{~d}$ inverse energy cascade, one derives in a similar way: $\left\langle\boldsymbol{v}(\boldsymbol{r}) p(\boldsymbol{r}) v^{2}(0)\right\rangle=\epsilon_{4} \boldsymbol{r} / 2$ where $\epsilon_{4}$ is the dissipation rate of the squared energy due to a large-scale sink. For the direct cascade of vorticity $\omega=\nabla \times \boldsymbol{v}$ in $2 \mathrm{~d}$, analogous flux relations on the correlation functions $\left\langle\boldsymbol{v}(\boldsymbol{r}) \omega^{n}(\boldsymbol{r}) \omega^{n}(0)\right\rangle$ do not contain pressure and were derived and analyzed by [8].

Let us discuss the validity limits of (16) where we neglected cumulants like the structure function $\left\langle\boldsymbol{u} u^{4}\right\rangle_{c}$. Such structure function scales as $r^{\zeta_{5}}$ and is subleading at sufficiently small $r$ since $\zeta_{5}>1$. In other words, there exists the scale $\ell$, below which the decoupling is possible. We ask now how $\ell$ and $\zeta_{5}$ may depend on the only parameter that enters Navier-Stokes equation, the space dimensionality $d$. For $d=3$ we expect $\ell \simeq L$. Usually, in statistical physics [28] and passive scalar theory [3, 7, 9, 19] the statistics is getting closer to Gaussian and decoupling improves when $d$ grows. Somewhat counter-intuitively, one may expect a different behaviour in turbulence because of diminishing role of pressure. To understand how the pressure depends on $d$, consider how the identity $\Delta p=-\nabla_{i} v_{j} \nabla_{j} v_{i}$ behaves when $d$ increases while the velocity components are kept fixed. The Laplacian has $d$ terms of different signs. Assuming that in the limit $d \rightarrow \infty$ those terms can be considered independent, their sum grows like $\sqrt{d}$. The rhs contains $d^{2}$ terms and grows like $d$ so that the pressure is expected to grow as $p \propto \sqrt{d}$. This is slower than $v^{2} \propto d$ so that assuming $d$ large one may neglect the pressure contribution into the correlation functions. Consider, for instance, the 
tensor of the fourth moment of the velocity difference:

$$
\begin{aligned}
& \left\langle u_{i} u_{j} u_{k} u_{l}\right\rangle=A(r) \hat{r}_{i} \hat{r}_{j} \hat{r}_{k} \hat{r}_{l}+C(r)\left[\delta_{i j} \delta_{k l}+\delta_{i k} \delta_{j l}+\delta_{i l} \delta_{j k}\right] \\
& +B(r)\left[\delta_{i j} \hat{r}_{k} \hat{r}_{l}+\delta_{i k} \hat{r}_{j} \hat{r}_{l}+\delta_{i l} \hat{r}_{k} \hat{r}_{j}+\delta_{j l} \hat{r}_{k} \hat{r}_{i}+\delta_{j k} \hat{r}_{i} \hat{r}_{l}+\delta_{k l} \hat{r}_{i} \hat{r}_{j}\right] .
\end{aligned}
$$

At $d \rightarrow \infty$ the difference between longitudinal and transversal velocity components is expected to decrease, as follows from the analysis of the second moment where incompressibility and isotropy requires $\left\langle u_{\perp}^{2}-u_{l}^{2}\right\rangle=r\left\langle u_{l}^{2}\right\rangle^{\prime} /(d-1)$. Comparing $\left\langle u_{l}^{4}\right\rangle=A+6 B+3 C$ with $\left\langle u_{\perp}^{4}\right\rangle=3 C$, we see that $A, B \ll C$ at $d \rightarrow \infty$. Now, we generalize for $d$ dimensions the relation between the velocity and pressure correlation functions, derived by [17] for three dimensions, and remarkably find out that $C$-terms cancel out:

$$
\left\langle p^{2}\right\rangle=\frac{d^{2}-1}{12} \int_{0}^{\infty} A\left(r^{\prime}\right) \frac{d r^{\prime}}{r^{\prime}}-\frac{d-1}{3} \int_{0}^{\infty} \frac{d r^{\prime}}{r^{\prime}}\left[A\left(r^{\prime}\right)+3 B\left(r^{\prime}\right)\right] .
$$

That means that indeed $\left\langle p^{2}\right\rangle$ grows slower than $\left\langle u^{4}\right\rangle$ as $d \rightarrow \infty$. Remind that generally the correlations functions containing $p$ and $u$ may scale differently yet considering the limit $d \rightarrow \infty$ at fixed $r$ one may neglect the pressure term in (15). That would give

$$
\nabla_{i}\left\langle v_{i}(\boldsymbol{r}) v^{2}(\boldsymbol{r}) v^{2}(0)\right\rangle=2\left\langle\boldsymbol{f} \cdot \boldsymbol{v} v^{2}\right\rangle-2 \epsilon\left\langle v^{2}\right\rangle=4 \epsilon\left\langle v^{2}\right\rangle / d
$$

(for Gaussian pumping) which is much different from $\epsilon\left\langle v^{2}\right\rangle$ given by (16). That means that at $d \rightarrow \infty$ there may exist an interval of scales, $L \gg r \gg \ell$, where cumulants are comparable to the reducible part so that $\ell$ is a crossover scale between (17) and (22). That would mean that $\ell$ decreases with $d$ while $\zeta_{5} \rightarrow 1$ as $d \rightarrow \infty$. Moreover, at $d \rightarrow \infty$, the same analysis can be done for all odd moments: $\zeta_{n}=1$ for $n \geq 1$. We thus come to the suggestion that the scaling of incompressible turbulence in the limit $d \rightarrow \infty$ may be the same as the scaling of Burgers turbulence. This would not be that surprising since a single incompressibility condition imposed on $d$ velocity components is expected to be less restrictive as $d$ grows, so that flow configurations close to shocks give the main contribution to the moments. Note that for shock-like configurations, one cannot decouple $\mathbf{u}$ and $\boldsymbol{V}$ as done in deriving (17). Technically, neglecting pressure, makes all quantities $\int v^{2 n} d \mathbf{r}$ to be integrals of motion of the Euler equation so that the linear scaling of all odd velocity moments express the constancy of fluxes of these integrals of motion, like in Burgers, see e.g. [1]. Holder inequality then requires the linear scaling for even moments too, which corresponds to an extreme nonGaussianity of the small-scale velocity statistics. Physically, pressure is a non-local field 
which couples different regions in space and is expected to act like "an intermittency killer" as remarked by [20], see also [5, 24]. One way to find the exponents at finite $d$ may be a large- $d$ expansion, which must thus be very different from that used by [3, 7, 28]: one needs to start here from a Burgers-like limit rather than from a Gaussian statistics. That explains, in particular, why no substantial simplification was found and why pressure terms were not small in the large- $d$ perturbation theory that starts from a Gaussian statistics [11].

Needless to say that the tendency of the exponents to approach unity with $d$ growing remains purely hypothetical on that level of analysis. However, if true, it means that the degree of non-Gaussianity of the statistics of a single velocity component grows with $d$, which agrees with the numerical comparison between three and four dimensions made in the remarkable work by Gotoh and co-authors [6]. Scalar quantities made out of vector products contain the sum of $d$ terms; one cannot generally conclude whether their statistics gets closer to Gaussian as $d$ increases because of the competition between increasing non-Gaussianity of a single term and the averaging over $d$ terms.

To conclude, the formulas (11, 17, 18) is the main result of this work.

\section{Acknowledgments}

The work was supported by the Israeli Science Foundation, the Deutsch-Israelische Projektkooperation, the US-Israel Binational Science Foundation, the Minerva Foundation and the German-Israeli Foundation. G.F. thanks M. Nelkin, U. Frisch, T. Gotoh and G. Boffetta for useful remarks.

[1] Cardy, J., Falkovich, G. \& Gawedzki, K. 2008 Non-equilibrium Statistical Mechanics and Turbulence, LMS Lect Note Series 355. Cambridge Univ. Press.

[2] Chandrasekhar, S. 1951 The Invariant Theory of Isotropic Turbulence in MagnetoHydrodynamics. Proc. Roy. Soc. A 204, 435-449.

[3] Chertkov, M., Falkovich, G., Kolokolov, I. \& Lebedev, V. 1995 Normal and anomalous scaling of the fourth-order correlation function of a randomly advected passive scalar. Phys Rev E $\mathbf{5 2}$ 4924-41.

[4] Chkhetiani, O. 1996 On the third moments in helical turbulence. JETP Lett. 63, 808-812. 
[5] Gotoh, T. \& Nakano, T. 2003 Role of Pressure in Turbulence J Stat. Phys. 113 855-874.

[6] Gotoh, T. et al. 2007 Statistical properties of four-dimensional turbulence. Phys. Rev. E 75, 016310.

[7] Falkovich, G., Kazakov, V. \& Lebedev, V. 1998 Particle dispersion in a multidimensional random flow with arbitrary temporal correlations. Physica A 249 (1-4) 36-46.

[8] Falkovich, G. \& Lebedev, V. 1994 Universal direct cascade in two-dimensional turbulence. Phys. Rev. E 50 3883-3899.

[9] Falkovich, G., Gawedzki, K. \& Vergassola, M. (2001) Particles and fields in fluid turbulence. Rev. Mod. Phys. 73 913-975.

[10] Falkovich, G. \& Sreenivasan, K. R. 2006 Lessons from hydrodynamic turbulence. Phys. Today 59 (4), 43-49.

[11] Fournier, J-D., Frisch, U. \& Rose, H. 1978 Infinite-dimensional turbulence. J. Phys. A 11, 187-198.

[12] Frisch, U. 1995 Turbulence: The Legacy of A. N. Kolmogorov. Cambridge University Press.

[13] Forster, D. 1975 Hydrodynamic Fluctuations, Broken Symmetry, And Correlation Functions, Perseus Books.

[14] Galtier, S. 2008 von Karman-Howarth equations for Hall magnetohydrodynamic flows. Phys. Rev. E 77, 015302(R).

[15] Gomez, T., Politano, H. \& Pouquet, A. 2000 An exact relationship for thirdorder structure functions in helical flows. Phys. Rev. E 61, 5321-5325.

[16] Gawedzki, K. 1999 Easy turbulence. chao-dyn/9907024.

[17] Hill, R. J. \& Wilczak, J. M. 1995 Pressure structure functions and spectra for locally isotropic turbulence. J Fluid Mech. 296, 247-269.

[18] Kolmogorov, A. N. 1941 Dissipation of energy in locally isotropic turbulence. Dokl. Akad. Nauk SSSR 3216.

[19] Kraichnan, R. H. 1974 Convection of a passive scalar by a quasi-uniform random straining field. J. Fluid Mech. 64, 737-762.

[20] Kraichnan, R. H. 1991 Turbulent cascade and intermittency growth. Proc. R. Soc. Lond. A 434:65.

[21] Kritsuk, A. G. et al. (2007) The Statistics of Supersonic Isothermal Turbulence. Astrophys. J 665 (1), 416-431. 
[22] Landau, L. \& Lifshits, E. 1987 Fluid Mechanics. Pergamon Press, Oxford.

[23] L'vov, V., Podivilov, E. \& Procaccia, I. (1997) Exact Result for the 3rd Order Correlations of Velocity in Turbulence with Helicity. http://xxx.lanl.gov/abs/chao-dyn/9705016

[24] Nelkin, M. 1975 Scaling theory of hydrodynamic turbulence. Phys. Rev. A 11, 1737-1743.

[25] Politano, H. \& Pouquet, A. 1998 von Karman-Howarth equation for magnetohydrodynamics and its consequences on third-order longitudinal structure and correlation functions. Phys. Rev. E 57, R21-R24.

[26] Podesta, J. J., Forman, M. A. \& Smith, C. W. 2007 Anisotropic form of third-order moments and relationship to the cascade rate in axisymmetric magnetohydrodynamic turbulence. Phys. of Plasmas 14, 092305.

[27] Podesta, J. J. 2008 Laws for third-order moments in homogeneous anisotropic incompressible magnetohydrodynamic turbulence. J. Fluid Mech. 609, 171-194.

[28] Stanley, H.E 1968 Spherical Model as the Limit of Infinite Spin Dimensionality. Phys. Rev. 176, 718-722.

[29] Yaglom, A. M. 1949 On the local structure of a temperature field in a turbulent flow. Dokl. Akad. Nauk. SSSR 69, 743.

[30] Yakhot, V. 2001 Mean-field approximation and a small parameter in turbulence theory. Phys. Rev. E 63, 026307. 